\title{
Bifurcations of a Ratio-Dependent Holling-Tanner System with Refuge and Constant Harvesting
}

\author{
Xia Liu ${ }^{1}$ and Yepeng Xing ${ }^{2}$ \\ ${ }^{1}$ College of Mathematics and Information Science, Henan Normal University, Xinxiang 453007, China \\ ${ }^{2}$ College of Mathematics and Science, Shanghai Normal University, Shanghai 200234, China \\ Correspondence should be addressed to Xia Liu; liuxiapost@163.com
}

Received 2 December 2012; Revised 10 January 2013; Accepted 13 January 2013

Academic Editor: Dragoş-Pătru Covei

Copyright (c) 2013 X. Liu and Y. Xing. This is an open access article distributed under the Creative Commons Attribution License, which permits unrestricted use, distribution, and reproduction in any medium, provided the original work is properly cited.

\begin{abstract}
The bifurcation properties of a predator prey system with refuge and constant harvesting are investigated. The number of the equilibria and the properties of the system will change due to refuge and harvesting, which leads to the occurrence of several kinds bifurcation phenomena, for example, the saddle-node bifurcation, Bogdanov-Takens bifurcation, Hopf bifurcation, backward bifurcation, separatrix connecting a saddle-node and a saddle bifurcation and heteroclinic bifurcation, and so forth. Our main results reveal much richer dynamics of the system compared to the system with no refuge and harvesting.
\end{abstract}

\section{Introduction}

The Holling-Tanner predator-prey system has attracted much attentions from both theoretical and mathematical biologists, especially, in [1] the authors considered the ratio-dependent system of the form

$$
\begin{gathered}
\dot{x}=r x\left(1-\frac{x}{K}\right)-\frac{\alpha x y}{A y+x}, \\
\dot{y}=s y\left(1-\frac{b y}{x}\right),
\end{gathered}
$$

where $x$ and $y$ stand for prey and predator population (or densities) at time $t$, respectively. The predator growth is of logistic type with growth rate $r$ and carrying capacity $K$ in the absence of predation; $\alpha$ and $A$ stand for the predator capturing rate and half saturation constant, respectively; $s$ is the intrinsic growth rate of predator; however, carrying capacity $x / b$ ( $b$ is the conversion rate of prey into predators) is the function on the population size of prey. They studied the global properties and the existence and uniqueness of limit cycle for system (1).

Generally speaking, from the views of the optimal management and exploitation of bioeconomic resources, it is necessary and meaningful to consider the refuge or harvesting of populations in some bioeconomic models; one can see [2-11], and the references therein.

In this paper we will analyze the system (1) with refuge and harvesting of the form

$$
\begin{gathered}
\dot{x}=r x\left(1-\frac{x}{K}\right)-\frac{\alpha y(x-\bar{m})}{A y+x-\bar{m}}-\bar{h}, \\
\dot{y}=s y\left(1-\frac{b y}{x-\bar{m}}\right),
\end{gathered}
$$

where $r, K, \alpha, A, \bar{m}, \bar{h}, s$, and $b$ are positive constants. $\bar{m}$ is a constant number of prey using refuges, and $\bar{h}$ is the rate of prey harvesting.

For simplicity, we first rescale the system (2).

Let $X=x-\bar{m}, Y=y$; system (2) can be written as (still denote $X, Y$ as $x, y$ )

$$
\begin{gathered}
\dot{x}=r(x+\bar{m})\left(1-\frac{x+\bar{m}}{K}\right)-\frac{\alpha x y}{A y+x}-\bar{h}, \\
\dot{y}=s y\left(1-\frac{b y}{x}\right) .
\end{gathered}
$$


Next, let $\tau=r t, X=x / K$, and $Y=\alpha y / r K$, then system (3) takes the form (still denote $X, Y$, and $\tau$ as $x, y, t$ )

$$
\begin{gathered}
\dot{x}=(x+m)(1-x-m)-\frac{x y}{a y+x}-h=P(x, y), \\
\dot{y}=\delta y\left(\beta-\frac{y}{x}\right)=Q(x, y),
\end{gathered}
$$

where $m=\bar{m} / K, a=A r / \alpha, \delta=s \bar{h} / \alpha, \beta=\alpha / b r$, and $h=\bar{h} / r$.

From the view of biology, we are only interested in the dynamics of the system (4) in the first quadrant.

The organization of this paper is as follows. In Section 2, we discuss the existence and properties of the equilibria of system (4). In Section 3, all possible bifurcation phenomena of the model in terms of the five parameters are presented, and the numerical simulations about every bifurcation phenomena are exhibited.

\section{Qualitative Analysis of Equilibria}

To obtain the boundary equilibria the following equation can be obtained

$$
x^{2}+(2 m-1) x+h-m(1-m)=0 .
$$

Its discriminant is $\Delta_{0}=1-4 h$.

Obviously, $\Delta_{0} \geq 0$ if $0<h \leq 1 / 4$ and $\Delta_{0}<0$ if $h>1 / 4$.

Hence, (5) has two distinct positive solutions $x_{01}=(1-$ $2 m+\sqrt{1-4 h}) / 2, x_{02}=(1-2 m-\sqrt{1-4 h}) / 2$ if $0<m<$ $1 / 2, m(1-m)<h<1 / 4$, a positive solution $x_{01}$ if $0<m<$ $1,0<h<m(1-m)$, a double solution $\bar{x}=(1-2 m) / 2>0$ if $0<m<1 / 2, h=1 / 4$, and a solution $x_{03}=1-2 m$ when $h=m(1-m)$ and $0<m<1 / 2$.

One can obtain the positive equilibrium of (4) by solving the equation

$$
x^{2}+\left(\frac{\beta}{a \beta+1}+2 m-1\right) x+h+m(m-1)=0 .
$$

We can derive that system (4) has two positive equilibria $P_{1}=\left(x_{1}, y_{1}\right)$ and $P_{2}=\left(x_{2}, y_{2}\right)$ if

$$
\begin{gathered}
0<m<\frac{1}{2}\left(1-\frac{\beta}{a \beta+1}\right), \\
m(1-m)<h<\frac{1}{4}\left(\frac{\beta}{a \beta+1}-1\right)^{2}+\frac{m \beta}{a \beta+1},
\end{gathered}
$$

where

$$
\begin{aligned}
x_{i}= & -\frac{1}{2}\left(\frac{\beta}{a \beta+1}+2 m-1\right) \\
& +(-1)^{i+1} \frac{\sqrt{\Delta}}{2}, \quad y_{i}=\beta x_{i}, \quad i=1,2, \\
\Delta= & \left(\frac{\beta}{a \beta+1}-1\right)^{2}+\frac{4 m \beta}{a \beta+1}-4 h .
\end{aligned}
$$

Moreover, we can show that system (4) just exists one positive equilibrium $P_{1}$ if $0<h<m(1-m)$ and $0<m<1$.
The positive equilibrium $P_{3}=\left(x_{3}, y_{3}\right)\left(P_{*}=\left(x_{*}, y_{*}\right)\right)$ of system (4) exists if $0<m<(1 / 2)(1-\beta /(a \beta+1))$, and $h=m(1-m)(0<m<(1 / 2)(1-\beta /(a \beta+1)), h=$ $\left.(1 / 4)(\beta /(a \beta+1)-1)^{2}+m \beta /(a \beta+1)\right)$, where $x_{3}=1-2 m-$ $\beta /(a \beta+1), y_{3}=\beta x_{3}, x_{*}=-(1 / 2)(\beta /(a \beta+1)+2 m-1)$, and $y_{*}=\beta x_{*}$.

Summarizing the previous discussion, the number and location of equilibria of system (4) can be described by the following lemmas.

Lemma 1. Let $1 / 2 \leq m<1$.

(i) System (4) has no equilibria when $h \geq m(1-m)$;

(ii) System (4) exist two equilibria $E_{1}=\left(x_{01}, 0\right)$ and $P_{1}$ when $0<h<m(1-m)$.

Lemma 2. Let $0<(1 / 2)(1-\beta /(a \beta+1)) \leq m<1 / 2$.

(i) System (4) has no equilibria when $h>1 / 4$.

(ii) System (4) has a unique equilibrium $\bar{E}=(\bar{x}, 0)$ when $h=1 / 4$.

(iii) System (4) has two equilibria $E_{1}=\left(x_{01}, 0\right)$ and $E_{2}=$ $\left(x_{02}, 0\right)$ when $m(1-m)<h<1 / 4$.

(iv) System (4) has an equilibrium $E_{3}=\left(x_{03}, 0\right)$ when $h=$ $m(1-m)$.

(v) System (4) has two equilibria $E_{1}$ and $P_{1}$ when $0<h<$ $m(1-m)$.

Lemma 3. Let $0<m<(1 / 2)(1-\beta /(a \beta+1))$ and $\widetilde{h}=$ $(1 / 4)(\beta /(a \beta+1)-1)^{2}+m \beta /(a \beta+1)$.

(i) System (4) has no equilibria when $h>1 / 4$.

(ii) System (4) has a unique equilibrium $\bar{E}=(\bar{x}, 0)$ when $h=1 / 4$.

(iii) System (4) has two equilibria $E_{1}$ and $E_{2}$ when $\widetilde{h}<h<$ $1 / 4$.

(iv) System (4) has three equilibria $E_{1}, E_{2}$, and $P_{*}$ when $h=$ $\widetilde{h}$.

(v) System (4) has four equilibria $E_{1}, E_{2}, P_{1}$, and $P_{2}$ when $m(1-m)<h<\tilde{h}$.

(vi) System (4) has two equilibria $E_{1}$ and $P_{1}$ when $0<h<$ $m(1-m)$.

(vii) System (4) has two equilibria $E_{3}$ and $P_{3}$ when $h=$ $m(1-m)$.

Next we discuss the dynamics of system (4) in the neighborhood of each feasible equilibria. Firstly, the Jacobian matrix of system (4) at $E_{1}$ is

$$
M_{E_{1}}=\left[\begin{array}{cc}
-\sqrt{1-4 h} & -1 \\
0 & \delta \beta
\end{array}\right] \text {. }
$$

It is easy to see that $E_{1}$, if exists, is a hyperbolic saddle.

Secondly, the Jacobian matrix of system (4) at $E_{2}$ is

$$
M_{E_{2}}=\left[\begin{array}{cc}
\sqrt{1-4 h} & -1 \\
0 & \delta \beta
\end{array}\right] .
$$


One can see that boundary equilibrium $E_{2}$, if exists, is an unstable hyperbolic node.

The Jacobian matrix of system (4) at $E_{3}$ is

$$
M_{E_{3}}=\left[\begin{array}{cc}
2 m-1 & -1 \\
0 & \delta \beta
\end{array}\right] \text {. }
$$

Hence, $E_{3}$, if exists, is a saddle.

Similarly, we assume boundary equilibrium $\bar{E}$ exists, and the Jacobian matrix of system (4) at $\bar{E}$ is obtained as follows:

$$
M_{\bar{E}}=\left[\begin{array}{cc}
0 & -1 \\
0 & \delta \beta
\end{array}\right] \text {. }
$$

Hence, $\bar{E}$ is a saddle node.

The previous discussion can be summarized as follows.

Theorem 4. If the equilibria $E_{1}, E_{2}$, and $E_{3}$ exist, then $E_{1}$ and $E_{3}$ are hyperbolic saddle, and $E_{2}$ is a hyperbolic unstable node. Moreover, $E_{1}$ and $E_{2}$ merge into a saddle node $\bar{E}=(\bar{x}, 0)$ when $h=1 / 4$.

Remark 5. Note that if $h=1 / 4$, then $\dot{x}=-(x+m-1 / 2)^{2}-$ $x y /(x+a y)<0$, if $h>1 / 4$, then $\dot{x} \leq 1 / 4-h<0$. Thus, the prey species may go extinct as time increases for some initial values when $h \geq 1 / 4$. That is, biological over harvesting occurs.

In the following, we will discuss the properties of interior equilibria of system (4).

2.1. The Properties of Interior Equilibria. The Jacobian matrix of system (4) at $P_{1}$ is

$$
M_{P_{1}}=\left[\begin{array}{c}
\frac{\beta}{(a \beta+1)^{2}}-\sqrt{\Delta}-\frac{1}{(a \beta+1)^{2}} \\
\delta \beta^{2}
\end{array}\right] .
$$

The characteristic equation is $\lambda^{2}+A_{1} \lambda+A_{2}=0$, where

$$
A_{1}=\sqrt{\Delta}-\frac{\beta}{(1+a \beta)^{2}}+\delta \beta, \quad A_{2}=\delta \beta \sqrt{\Delta}>0 .
$$

Denote that

$$
\begin{aligned}
A_{1}^{2}-4 A_{2}= & \beta^{2} \delta^{2}-2\left(\sqrt{\Delta}+\frac{\beta}{(1+a \beta)^{2}}\right) \beta \delta \\
& +\left(\sqrt{\Delta}-\frac{\beta}{(1+a \beta)^{2}}\right)^{2}:=F(\delta) .
\end{aligned}
$$

The discriminant of $F(\delta)=0$ is $\Delta_{1}=16 \beta^{3} \sqrt{\Delta} /(1+a \beta)^{2}>0$, then $F(\delta)=0$ has two distinct solutions $\delta_{1}$ and $\delta_{2}$ denoted by

$$
\delta_{1}=\left(\sqrt{\frac{\sqrt{\Delta}}{\beta}}-\frac{1}{1+a \beta}\right)^{2}, \quad \delta_{2}=\left(\sqrt{\frac{\sqrt{\Delta}}{\beta}}+\frac{1}{1+a \beta}\right)^{2} .
$$

If $\sqrt{\Delta} \neq \beta /(1+a \beta)^{2}$, it is easy to see that $P_{1}$ is a node if $0<$ $\delta<\delta_{1}$ or $\delta>\delta_{2}$, a degenerate node if $\delta=\delta_{1}$ or $\delta=\delta_{2}$, and a focus or a center type nonhyperbolic if $\delta_{1}<\delta<\delta_{2}$.

If $\sqrt{\Delta}=\beta /(1+a \beta)^{2}, P_{1}$ is a node if $\delta>\delta_{2}$, and a degenerate node if $\delta=\delta_{2}$, and a focus or a center-type nonhyperbolic if $0<\delta<\delta_{2}$.

To discuss the stability of $P_{1}$, we need to determine the sign of $A_{1}$. Define $\widetilde{\delta}=1 /(1+a \beta)^{2}-(1 / \beta) \sqrt{\Delta}$, then $A_{1}=$ $\beta(\delta-\widetilde{\delta})$.

Clearly, if $\beta /(1+a \beta)^{2} \leq \sqrt{\Delta}$ then $A_{1}>0$ for all $\delta$; if $\beta /(1+a \beta)^{2}>\sqrt{\Delta}$, then $A_{1}>0$ when $\delta>\widetilde{\delta}, A_{1} \leq 0$ when $0<\delta \leq \widetilde{\delta}$; by simple computation, one can obtain $F(\widetilde{\delta})=$ $4 \sqrt{\Delta}\left(\sqrt{\Delta}-\beta /(a \beta+1)^{2}\right)<0$, hence $\delta_{1}<\widetilde{\delta}<\delta_{2}$.

The Jacobian matrix of system (4) at $P_{2}$ is

$$
M_{P_{2}}=\left[\begin{array}{cc}
\frac{\beta}{(a \beta+1)^{2}}+\sqrt{\Delta} & -\frac{1}{(a \beta+1)^{2}} \\
\delta \beta^{2} & -\delta \beta
\end{array}\right] .
$$

Its determinant is $\operatorname{det} M_{P_{2}}=-\delta \beta \sqrt{\Delta}<0$.

Through the previous discussion, about the stability of $P_{1}$ and $P_{2}$, we have the following theorem.

Theorem 6. Equilibrium $P_{2}$, if exists, must be a hyperbolic saddle. Equilibrium $P_{1}$, if exists, may be a node or a focus when $\beta /(1+a \beta)^{2} \leq \sqrt{\Delta}$. And when $\beta /(1+a \beta)^{2}>\sqrt{\Delta}, P_{1}$ is a stable focus for $\delta_{2}>\delta>\widetilde{\delta}$, a stable degenerate node for $\delta=\delta_{2}$, a stable node for $\delta>\delta_{2}$, an unstable node for $0<\delta<\delta_{1}$, an unstable degenerate node for $\delta=\delta_{1}$, an unstable focus for $\delta_{1}<\delta<\widetilde{\delta}$, and a weak focus or a center for $\delta=\widetilde{\delta}$.

The Jacobian matrix of system (4) at $P_{3}$ is

$$
M_{P_{3}}=\left[\begin{array}{cc}
2 m-1+\frac{\beta(a \beta+2)}{(a \beta+1)^{2}} & -\frac{1}{(a \beta+1)^{2}} \\
\delta \beta^{2} & -\delta \beta
\end{array}\right] .
$$

then by the existence condition of $P_{3}$, det $M_{P_{3}}=-\beta \delta(2 m-$ $1+\beta /(a \beta+1))>0, \operatorname{tr} M_{P_{3}}=2 m-1-\delta \beta+\beta /(1+$ $a \beta)+\beta /(1+a \beta)^{2}$. Then by taking similar methods used in estimating the properties of $P_{1}$, we have the following theorem.

Theorem 7. Let $h=m(1-m), \bar{\delta}=(1 / \beta)(2 m-1+\beta /(1+$ $\left.a \beta)+\beta /(1+a \beta)^{2}\right)$. Then,

(i) assume $0<m \leq(1 / 2)\left(1-\beta /(1+a \beta)-\beta /(1+a \beta)^{2}\right)$, then $P_{3}$ is stable;

(ii) assume $(1 / 2)\left(1-\beta /(1+a \beta)-\beta /(1+a \beta)^{2}\right)<m<$ $(1 / 2)(1-\beta /(1+a \beta))$, then $P_{3}$ is stable if $\delta>\bar{\delta}$, is unstable if $0<\delta<\bar{\delta}$, and is a weak focus or a center if $\delta=\bar{\delta}$.

The Jacobian matrix of system (4) at $P_{*}$ is

$$
M_{P_{*}}=\left[\begin{array}{cc}
\frac{\beta}{(a \beta+1)^{2}} & -\frac{1}{(a \beta+1)^{2}} \\
\delta \beta^{2} & -\delta \beta
\end{array}\right] .
$$


One can see that $\operatorname{det} M_{P_{*}}=0$, which indicates that $P_{*}$ is a degenerate singularity and maybe has complicated properties, see the following theorem.

Theorem 8. Let $0<m<(1 / 2)(1-\beta /(a \beta+1)), h=\tilde{h}$. Then system (4) has three equilibria, where $E_{1}$ is a hyperbolic saddle, $E_{2}$ is a hyperbolic unstable node, and $P_{*}$ is a degenerate singularity. More precisely,

$1^{\circ}$ if $\delta \neq 1 /(a \beta+1)^{2}$, then $P_{*}$ is a saddle node;

$2^{\circ}$ if $\delta=1 /(a \beta+1)^{2}$, then $P_{*}$ is a cusp of codimension 2 .

Proof. In order to discuss the properties of system (4) in the neighborhood of the equilibrium $P_{*}=\left(x_{*}, y_{*}\right)$, we first take $\bar{x}=x-x_{*}, \bar{y}=y-y_{*}$, then $P_{*}$ is translated to $(0,0)$, and system (4) becomes (still denote $\bar{x}, \bar{y}$ as $x, y$ )

$$
\begin{aligned}
\dot{x}= & \frac{\beta}{(a \beta+1)^{2}} x-\frac{1}{(a \beta+1)^{2}} y-g_{1} x^{2} \\
& +g_{2} x y-g_{3} y^{2}+O\left(|x, y|^{3}\right), \\
\dot{y}= & \delta \beta^{2} x-\delta \beta y+g_{4} x^{2}-g_{5} x y+g_{6} y^{2}+O\left(|x, y|^{3}\right),
\end{aligned}
$$

where

$$
\begin{aligned}
& g_{1}=\frac{2 a \beta^{2}}{(a \beta+1)^{2}((2 m-1)(a \beta+1)+\beta)}+1, \\
& g_{2}=\frac{4 a \beta}{(a \beta+1)^{2}((2 m-1)(a \beta+1)+\beta)}, \\
& g_{3}=\frac{2 a}{(a \beta+1)^{2}((2 m-1)(a \beta+1)+\beta)}, \\
& g_{4}=\frac{2 \delta \beta^{2}(a \beta+1)}{(2 m-1)(a \beta+1)+\beta}, \\
& g_{5}=\frac{4 \delta \beta(a \beta+1)}{(2 m-1)(a \beta+1)+\beta}, \\
& g_{6}=\frac{2 \delta(a \beta+1)}{(2 m-1)(a \beta+1)+\beta} .
\end{aligned}
$$

Clearly, if $\delta \neq 1 /(a \beta+1)^{2}$, then $\operatorname{tr}\left(M_{P_{*}}\right) \neq 0 . P_{*}=\left(x_{*}, y_{*}\right)$ is a saddle-node. We finish the proof of the part $1^{\circ}$.

When $\delta=1 /(a \beta+1)^{2}, \operatorname{tr} M_{P_{*}}=0$, which implies that both eigenvalues of the matrix $M_{P_{*}}$ are zero. We rewrite system (20) as

$$
\begin{aligned}
\dot{x}= & \frac{\beta}{(a \beta+1)^{2}} x-\frac{1}{(a \beta+1)^{2}} y-q_{1} x^{2} \\
& +q_{2} x y-q_{3} y^{2}+O\left(|x, y|^{3}\right), \\
\dot{y}= & \frac{\beta^{2}}{(a \beta+1)^{2}} x-\frac{\beta}{(a \beta+1)^{2}} y+q_{4} x^{2} \\
& -q_{5} x y+q_{6} y^{2}+O\left(|x, y|^{3}\right),
\end{aligned}
$$

where

$$
\begin{aligned}
& q_{1}=g_{1}, \quad q_{2}=g_{2}, \quad q_{3}=g_{3}, \\
& q_{4}=\frac{2 \beta^{2}}{(a \beta+1)^{2}((2 m-1)(a \beta+1)+\beta)}, \\
& q_{5}=\frac{4 \beta}{(a \beta+1)^{2}((2 m-1)(a \beta+1)+\beta)}, \\
& q_{6}=\frac{2}{(a \beta+1)^{2}((2 m-1)(a \beta+1)+\beta)} .
\end{aligned}
$$

By introducing variable $\tau=\left(\beta /(a \beta+1)^{2}\right) t$ into previous system and rewriting $\tau$ as $t$ for simplicity, then we obtain that

$$
\begin{aligned}
& \dot{x}=x-\frac{1}{\beta} y-\omega_{1} x^{2}+\omega_{2} x y-\omega_{3} y^{2}+O\left(|x, y|^{3}\right), \\
& \dot{y}=\beta x-y+\omega_{4} x^{2}-\omega_{5} x y+\omega_{6} y^{2}+O\left(|x, y|^{3}\right),
\end{aligned}
$$

where

$$
\begin{aligned}
& \omega_{1}=\frac{2 a \beta}{(2 m-1)(a \beta+1)+\beta}+\frac{(a \beta+1)^{2}}{\beta}, \\
& \omega_{2}=\frac{4 a}{(2 m-1)(a \beta+1)+\beta}, \\
& \omega_{3}=\frac{2 a}{\beta((2 m-1)(a \beta+1)+\beta)}, \\
& \omega_{4}=\frac{2 \beta}{(2 m-1)(a \beta+1)+\beta}, \\
& \omega_{5}=\frac{4}{(2 m-1)(a \beta+1)+\beta}, \\
& \omega_{6}=\frac{2}{\beta((2 m-1)(a \beta+1)+\beta)} .
\end{aligned}
$$

We take transformation $X_{0}=x, Y_{0}=x-(1 / \beta) y$ into (24), then system (24) is transformed to

$$
\begin{aligned}
& \dot{X}_{0}=Y_{0}+\eta_{1} X_{0}^{2}+\eta_{2} X_{0} Y_{0}-\omega_{3} \beta^{2} Y_{0}^{2}+O\left(\left|X_{0}, Y_{0}\right|^{3}\right), \\
& \dot{Y}_{0}=\eta_{3} X_{0}^{2}+\eta_{4} X_{0} Y_{0}-\eta_{5} Y_{0}^{2}+O\left(\left|X_{0}, Y_{0}\right|^{3}\right),
\end{aligned}
$$

where

$$
\begin{aligned}
& \eta_{1}=-\omega_{3} \beta^{2}-\omega_{1}+\omega_{2} \beta, \\
& \eta_{2}=2 \omega_{3} \beta^{2}-\omega_{2} \beta, \\
& \eta_{3}=-\frac{\omega_{1} \beta-\omega_{2} \beta^{2}+\omega_{3} \beta^{3}+\omega_{4}-\omega_{5} \beta+\omega_{6} \beta^{2}}{\beta}, \\
& \eta_{4}=2 \omega_{3} \beta^{2}-\omega_{2} \beta-\omega_{5}+2 \omega_{6} \beta, \\
& \eta_{5}=\beta\left(\omega_{3} \beta+\omega_{6}\right) .
\end{aligned}
$$


In order to obtain the canonical normal forms of system (26), we will perform a series of $C^{\infty}$ transformations of variables for system (26) in a small neighborhood of $(0,0)^{\mathrm{T}}$.

Firstly, performing the transformation by taking $X_{1}=$ $X_{0}, Y_{1}=Y_{0}-\omega_{3} \beta^{2} Y_{0}^{2}$, then (26) becomes

$$
\begin{gathered}
\dot{X}_{1}=Y_{1}+\eta_{1} X_{1}^{2}+\eta_{2} X_{1} Y_{1}+O\left(\left|X_{1}, Y_{1}\right|^{3}\right), \\
\dot{Y}_{1}=\eta_{3} X_{1}^{2}+\eta_{4} X_{1} Y_{1}-\eta_{5} Y_{1}^{2}+O\left(\left|X_{1}, Y_{1}\right|^{3}\right) .
\end{gathered}
$$

Secondly, performing the transformation by taking $X_{2}=$ $X_{1}, Y_{2}=Y_{1}+\eta_{5} X_{1} Y_{1}$, then (28) becomes

$$
\begin{gathered}
\dot{X}_{2}=Y_{2}+\eta_{1} X_{2}^{2}+\left(\eta_{2}-\eta_{5}\right) X_{2} Y_{2}+O\left(\left|X_{2}, Y_{2}\right|^{3}\right), \\
\dot{Y}_{2}=\eta_{3} X_{2}^{2}+\eta_{4} X_{2} Y_{2}+O\left(\left|X_{2}, Y_{2}\right|^{3}\right) .
\end{gathered}
$$

We perform the final transformation of variables by

$$
X=X_{2}-\frac{\eta_{2}-\eta_{5}}{2} X_{2}^{2}, \quad Y=Y_{2}+\eta_{1} X_{2}^{2}+O\left(\left|X_{2}, Y_{2}\right|^{3}\right) .
$$

Then, we obtain

$$
\begin{gathered}
\dot{X}=Y, \\
\dot{Y}=\eta_{3} X^{2}+\left(2 \eta_{1}+\eta_{4}\right) X Y+O\left(|X, Y|^{3}\right) .
\end{gathered}
$$

Note that

$$
\eta_{3}=-\frac{(a \beta+1)^{2}}{\beta} \neq 0, \quad 2 \eta_{1}+\eta_{4}=-\frac{2(a \beta+1)^{2}}{\beta} \neq 0,
$$

which indicates that the origin $(0,0)$ of $(31)$ is a cusp of codimension 2. We complete the proof.

\section{Bifurcation Analysis}

From previous analysis, we can see the equilibria of system (4) may be hyperbolic or degenerate singularities under appropriate conditions, which indicate that some bifurcations may occur for system (4). It is interesting to investigate what kinds of bifurcations system (4) can undergo with the original parameters varying.

3.1. Hopf Bifurcation. Theorem 6 shows that $P_{1}$, if exists, is a weak focus or a center when

$$
\begin{aligned}
& V=\{(a, m, \beta, \delta, h): 0<m<\widetilde{m}, m(1-m) \\
& \left.<h<\tilde{h}, \frac{\beta}{(1+a \beta)^{2}}>\sqrt{\Delta}, \delta=\tilde{\delta}\right\},
\end{aligned}
$$

where $\widetilde{m}=(1 / 2)(1-\beta /(a \beta+1))$.

To determine the direction of Hopf bifurcation and stability of $P_{1}$ in this case, we need to compute the Liapunov coefficients of the equilibrium $P_{1}$. Let $\delta=\widetilde{\delta}$ and by the variable $u=x-x_{1}, v=y-y_{1}$. Then we rewrite system (4) (still denote $u, v$ as $x, y$ ) as follows:

$$
\begin{gathered}
\dot{x}=\tilde{\delta} \beta x-\frac{1}{(a \beta+1)^{2}} y+f_{1}(x, y), \\
\dot{y}=\widetilde{\delta} \beta^{2} x-\widetilde{\delta} \beta y+f_{2}(x, y) .
\end{gathered}
$$

We perform the transformations

$$
\begin{aligned}
& X=x, \quad Y=\widetilde{\delta} \beta x-\frac{1}{(a \beta+1)^{2}} y, \\
& u=X, \quad v=\frac{(a \beta+1) Y}{\beta \sqrt{\tilde{\delta}\left(1-\widetilde{\delta}(a \beta+1)^{2}\right)}}
\end{aligned}
$$

and rewrite $u, v$ as $x, y$. Then the previous system can be transformed to

$$
\begin{aligned}
\dot{x}= & k_{1} y+a_{20} x^{2}+a_{11} x y+a_{02} y^{2} \\
& +a_{30} x^{3}+a_{21} x^{2} y+a_{12} x y^{2}+a_{03} y^{3}+O\left(|(x, y)|^{4}\right), \\
\dot{y}= & -k_{1} x+d_{11} x y+b_{20} x^{2}+b_{11} x y+b_{02} y^{2} \\
& +b_{30} x^{3}+b_{21} x^{2} y+b_{12} x y^{2}+b_{03} y^{3}+O\left(|(x, y)|^{4}\right),
\end{aligned}
$$

where the expressions of $a_{20}, a_{11}, a_{02}, a_{30}, a_{21}, a_{12}$, $a_{03}, b_{20}, b_{11}, b_{02}, b_{30}, b_{21}, b_{12}$, and $b_{03}$ depend on the parameters $a, \beta, \delta, h$, and $m$, and $k_{1}=$

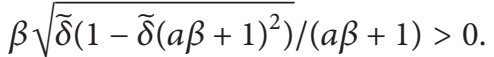

Using the formula, the first Liapunov number is

$$
\begin{gathered}
\sigma=-\frac{1}{8 \beta k_{1}}\left(\frac{2(a \beta+1)^{2}}{\widetilde{\delta}(a \beta+1)^{2}-1}+\frac{2 \beta(2 a \beta+1)}{(a \beta+1) x_{1}}\right. \\
\left.+\frac{a \beta^{3}(2 a \beta-1)\left[\widetilde{\delta}(a \beta+1)^{2}-1\right]}{x_{1}^{2}(a \beta+1)^{4}}\right) .
\end{gathered}
$$

Therefore, there exists a surface $H_{b}\left(H_{p}\right)$ in the parameter space $(a, m, \beta, \delta, h)$ which satisfies

$$
\begin{gathered}
H_{b}=\{(a, m, \beta, \delta, h): \sigma>0,(a, m, \beta, \delta, h) \in V\}, \\
\left(H_{p}=\{(a, m, \beta, \delta, h): \sigma<0,(a, m, \beta, \delta, h) \in V\}\right) .
\end{gathered}
$$

Hence, when the parameter $(a, m, \beta, \delta, h)$ is in $H_{b}\left(H_{p}\right)$, the equilibrium $P_{1}$ of system (4) is a weak focus of multiplicity 1 and is unstable (stable) (see [8]). $H_{b}\left(H_{p}\right)$ is called the subcritical (supercritical) Hopf bifurcation surface of system (4).

From Theorem 6, we know that $P_{1}$ is a stable focus for $\delta_{2}>$ $\delta>\widetilde{\delta}$ and $(a, m, \beta, \delta, h) \in V$, an unstable focus for $\delta_{1}<\delta<$ $\widetilde{\delta}$ and $(a, m, \beta, \delta, h) \in V$. 


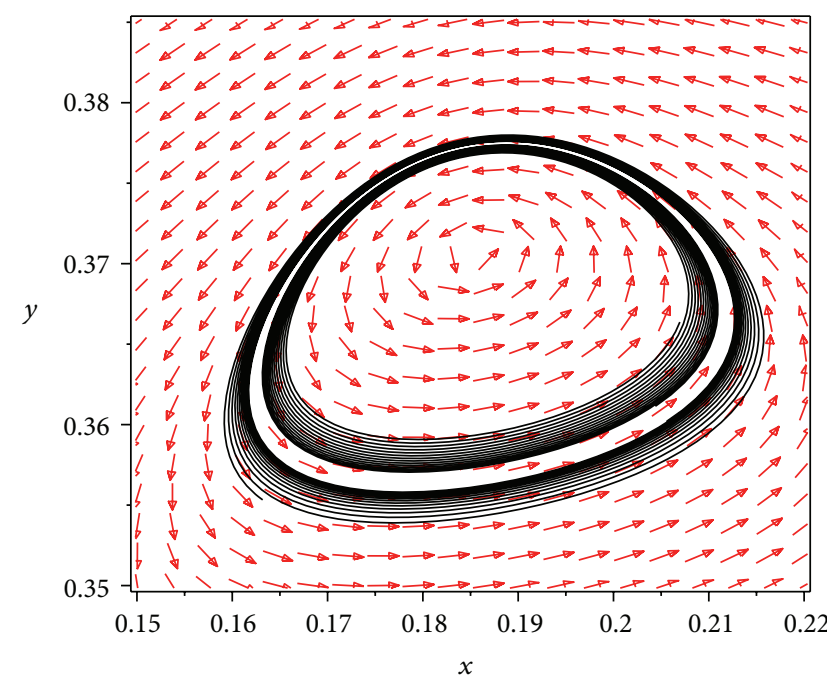

(a)

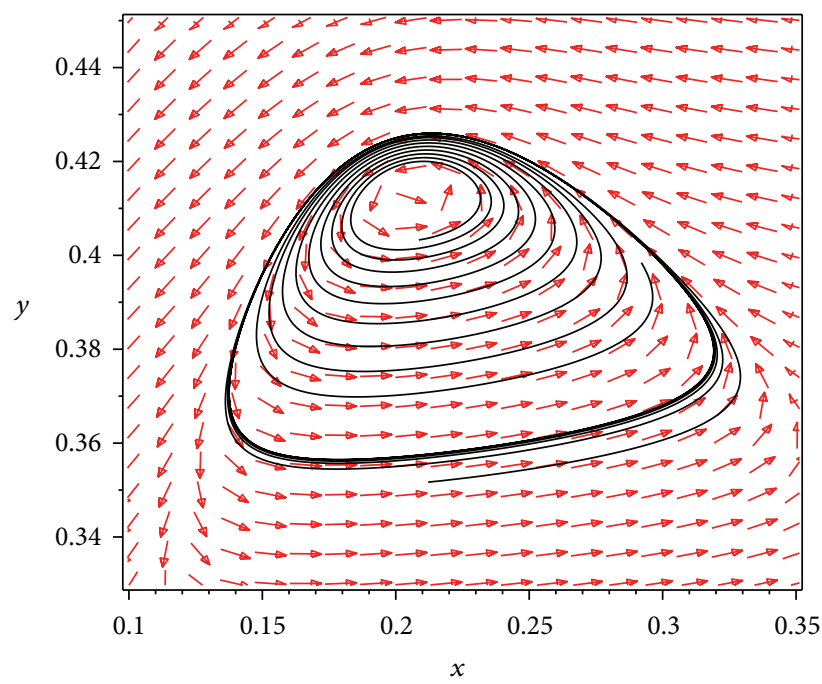

(b)

Figure 1: (a) System (4) shows an unstable limit cycle when $a=1.6, \beta=2, m=0.13095$, and $h=0.128 . \delta_{2} \approx 0.2220>\delta=0.00249>\delta \tilde{\delta} \approx$ 0.00235; (b) System (4) shows a stable limit cycle when $a=1.2, \beta=2, m=0.08235$, and $h=0.08396 . \delta_{1} \approx 0.0000391<\delta=0.002919<\widetilde{\delta} \approx$ 0.003639 .

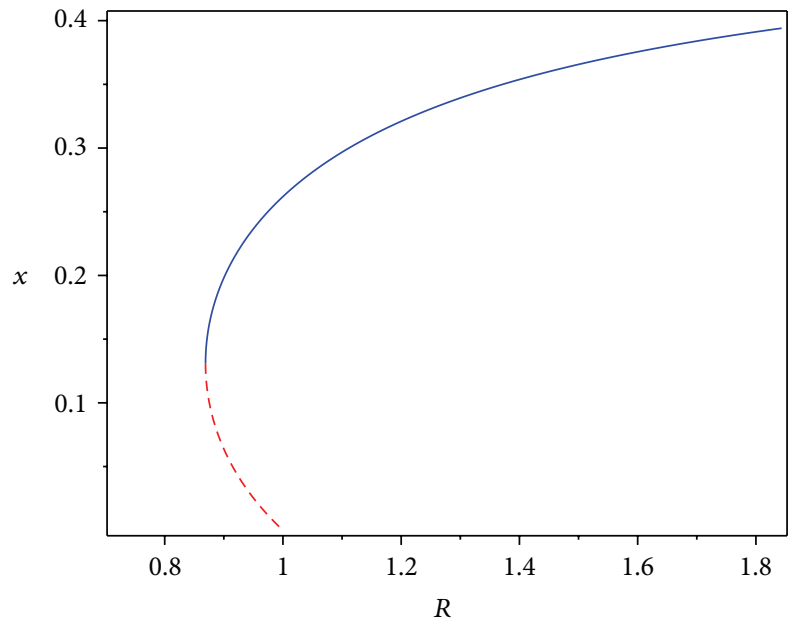

FIGURE 2: The figure of prey $x$ at equilibria versus $R$ when $a=1.6$, $\beta=2, m=0.1309$, and $h=0.13$, which displays a backward bifurcation at $R=1$.

Theorem 9. (i) System (4) has at least one unstable limit cycle if $(a, m, \beta, \delta, h) \in H_{b}, \delta_{2}>\delta>\widetilde{\delta},|\delta-\widetilde{\delta}| \ll 1$.

(ii) System (4) has at least one stable limit cycle if $(a, m, \beta, \delta, h) \in H_{p}, \delta_{1}<\delta<\widetilde{\delta},|\delta-\widetilde{\delta}| \ll 1$.

Remark 10. When $\sigma=0$ system (4) maybe undergoes degenerate Hopf bifurcation for some parameter values; since the expression of $\sigma$ is complicated, we do not discuss this case.

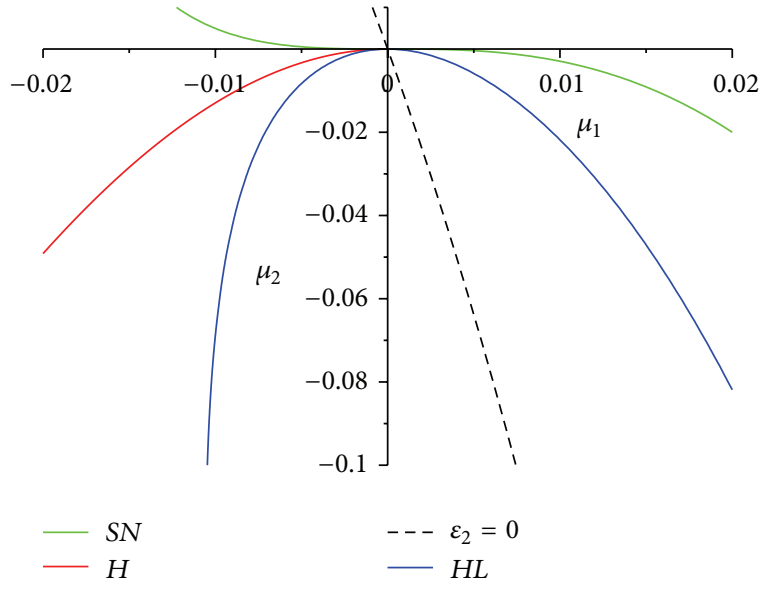

FIgURE 3: Bifurcation diagram of system (4) near $P_{*}$ in the plane of $\mu_{1}$ and $\mu_{2}$.

Note that by Theorem 7, if $P_{3}$ is a weak focus or a center, then we can obtain that its first Lyapunov number is

$$
\sigma_{1}=\frac{3 a \beta}{8 k_{1}(1+a \beta)(2 m a \beta-a \beta+\beta+2 m-1)}<0,
$$

therefore, $P_{3}$ is a stable weak focus.

By numerical calculation, we give the parameter values $(a, \beta, m, h)=(1.6,2.0,0.13095,0.128)$, then $\widetilde{\delta}=0.00235$, $\delta_{2}=0.2220$ and $k_{1} \sigma=0.31085>0$, and the existence condition of subcritical Hopf bifurcation is satisfied. If we 


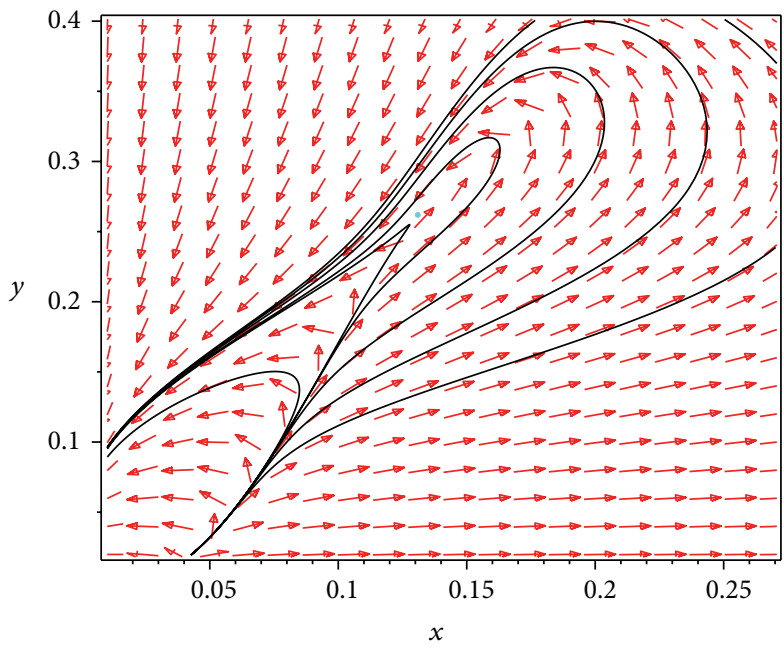

(a)

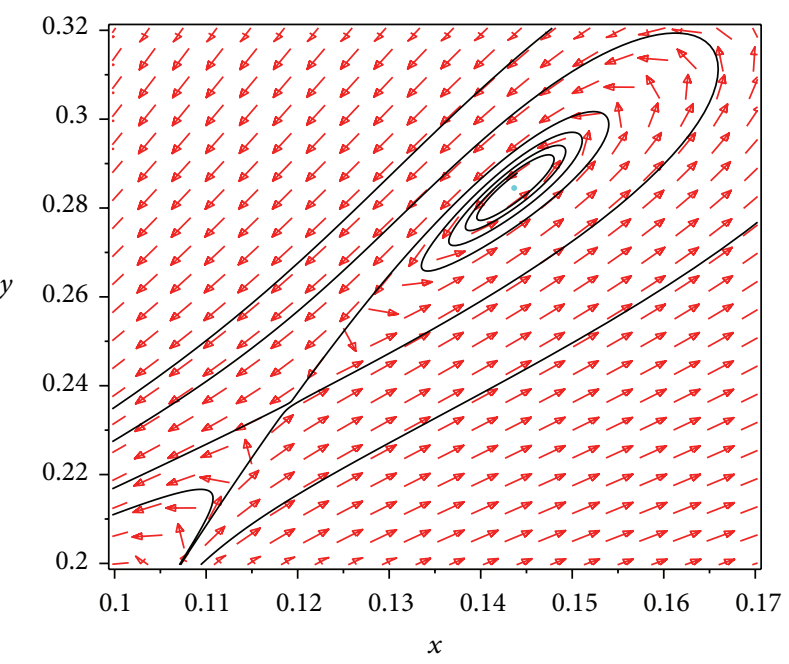

(b)

FIGURE 4: (a) System (4) shows a cusp of codimension 2 when $a=1.6, \beta=2, m=(1 / 4)(1-\beta /(a \beta+1)), h=(1 / 4)(\beta /(a \beta+1)-1)^{2}+$ $m \beta /(a \beta+1), \delta=1 /(a \beta+1)^{2}$, and $\mu_{1}=0, \mu_{2}=0$; (b) the cusp of codimension 2 break into an unstable focus $\widehat{E}_{1}$ and a hyperbolic saddle $\widehat{E}_{2}$ when $\mu_{1}=-0.0132, \mu_{2}=-0.02$.

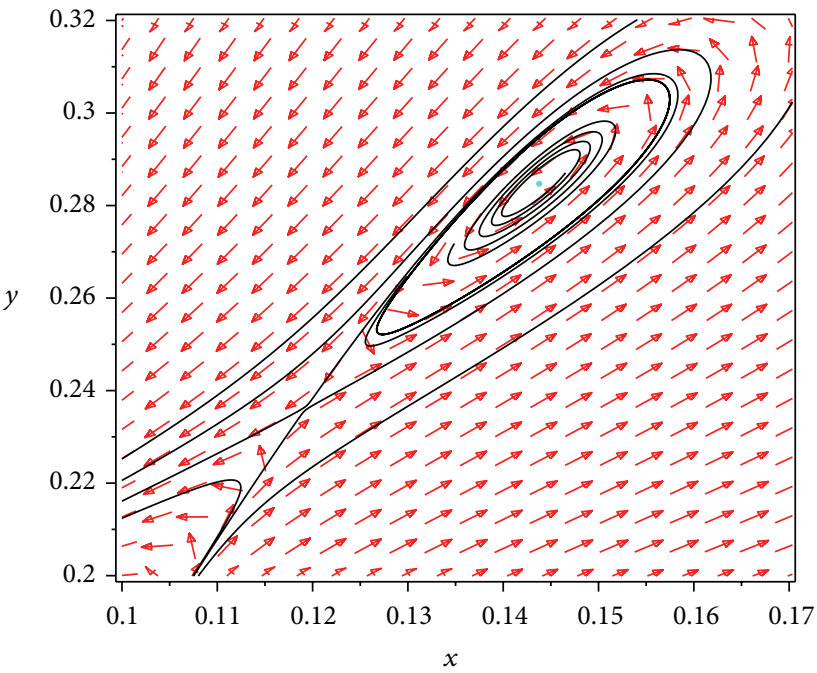

(a)

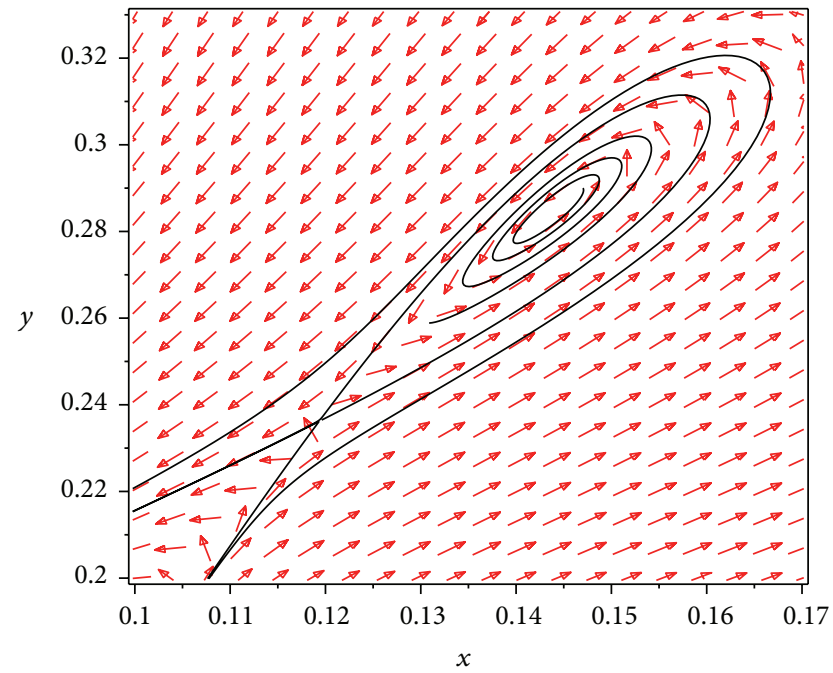

(b)

Figure 5: (a) The cusp of codimension 2 breaks into a stable focus $\widehat{E}_{1}$ and a hyperbolic saddle $\widehat{E}_{2}$ when $\mu_{1}=-0.0098, \mu_{2}=-0.02$. The change of stability of the focus yields an unstable limit cycle. (b) The unstable limit cycle is broken when $\mu_{1} \approx-0.0081768, \mu_{2}=-0.02$, reachs the manifold of the saddle $\widehat{E}_{2}$, and leads to a homoclinic loop occur.

keep $a, \beta, m, h$ fixed and choose $\delta=0.00249$, then a unstable limit cycle can be shown in Figure 1(a).

When taking $(a, \beta, m, h)=(1.2,2.0,0.08235,0.08396)$, then $\widetilde{\delta}=0.003639, \delta_{1}=0.0000391$, and $k_{1} \sigma=-0.1741543<$ 0 which satisfy the existence condition of supercritical Hopf bifurcation. Furthermore, we choose $\delta=0.002919$; according to Theorem 9, there exists a stable limit cycle, which can be shown in Figure 1(b).

3.2. Backward Bifurcation. Define $R=m(1-m) / h, R_{*}=$ $m(1-m) / \widetilde{h}$. 
Lemmas 2-3 and Theorems 6-8 illustrate that when the parameter $h$ varies in the range of $(0, m(1-m)]$, system (4) just has only one positive equilibrium $P_{1}$ which is stable. However, when $h$ varies in the range $(m(m-1), \widetilde{h})$, system $(4)$ has two distinct positive equilibria $P_{1}$ and $P_{2}$, where $P_{1}$ is a stable node or focus and $P_{2}$ is a saddle. Furthermore, when $h=\widetilde{h}$, system (4) has unique positive equilibrium $P_{*}$. The previous discussion indicates the possibility of a backward bifurcation, which can be summarized as follows.

Theorem 11. Let $0<m<(1 / 2)(1-\beta /(a \beta+1)), \delta>\delta_{2}$. Then system (4) has a unique positive equilibrium $P_{*}$ when $R=R_{*}$, has two distinct positive equilibria $P_{1}$ and $P_{2}$ when $R_{*}<R<$ 1 , where $P_{1}$ is a stable node and $P_{2}$ is a saddle, and has one positive equilibrium $P_{1}$ or $P_{3}$ when $R \geq 1$. Therefore, system (4) undergoes a backward bifurcation when $R=1$.

We give a numerical example in Figure 2 which displays that system (4) has a backward bifurcation at $R=1$.

3.3. Saddle-Node Bifurcations. From Lemmas 2-3 and Theorem 4 , we see that when $0<m<1 / 2, h=1 / 4, E_{1}$ and $E_{2}$ degenerate into a saddle-node $\bar{E}=(\bar{x}, 0)$. This indicates that there is a saddle node bifurcation surface which takes the form

$$
\begin{gathered}
\mathrm{SN}_{1}=\left\{(m, a, \beta, h, \delta): 0<m<\frac{1}{2}, h=\frac{1}{4}, \delta>0,\right. \\
\beta>0, a>0\} .
\end{gathered}
$$

Similarly, from Lemma 3 and the part $1^{\circ}$ of Theorem 8 , we know that when $0<m<(1 / 2)(1-\beta /(a \beta+1))$ and $h=\widetilde{h}$, in $\mathbb{R}_{2}^{+}$, system (4) admits the double point $P_{*}=\left(x_{*}, y_{*}\right)$. And $P_{*}$ is a saddle node if $\delta \neq 1 /(a \beta+1)^{2}$.

One also can see that when the parameter $h$ varies in the range of $(m(m-1), \widetilde{h})$, system (4) has two distinct positive equilibria $P_{1}$ and $P_{2}$. From Theorem 6, we know that $P_{1}$ may be a stable node, or a focus, and $P_{2}$ is a saddle. These imply that system (4) undergoes another saddle-node bifurcation of codimension 1. That is, there is a second saddle-node bifurcation surface $\mathrm{SN}_{2}$ which is defined by

$$
\begin{gathered}
\mathrm{SN}_{2}=\left\{(m, a, \beta, h, \delta): 0<m<\frac{1}{2}\left(1-\frac{\beta}{a \beta+1}\right),\right. \\
\left.h=\tilde{h}, \delta \neq \frac{1}{(a \beta+1)^{2}}\right\} .
\end{gathered}
$$

3.4. Bogdanov-Takens Bifurcation. From the part $2^{\circ}$ of Theorem 8, we can see that system (4) exists a cusp of codimension 2, which implies that there may exist the
Bogdanov-Takens bifurcation in system (4). Clearly, there exists a parameter space

$$
\begin{aligned}
& \mathrm{BT}=\{(m, a, \beta, h, \delta): \\
& 0<m<\frac{1}{2}-\frac{\beta}{2(a \beta+1)}, \delta=\frac{1}{(a \beta+1)^{2}}, \\
& \left.h=\frac{1}{4}\left(\frac{\beta}{a \beta+1}-1\right)^{2}+\frac{m \beta}{a \beta+1}\right\} \text {, }
\end{aligned}
$$

such that system (4) has a cusp of codimension 2 when $(m, a, \beta, h, \delta) \in \mathrm{BT}$.

To show that system (4) undergoes the Bogdanov-Takens bifurcation we choose $\delta$ and $\beta$ as bifurcation, parameters. We need to find the universal unfolding of $P_{*}$.

Let $(m, a, \beta, h, \delta) \in \mathrm{BT}$, and consider the following unfold system

$$
\begin{gathered}
\dot{x}=(x+m)(1-x-m)-\frac{y x}{a y+x}-h, \\
\dot{y}=\left(\delta+\mu_{1}\right) y\left(\beta+\mu_{2}-\frac{y}{x}\right),
\end{gathered}
$$

where $\mu_{1}$ and $\mu_{2}$ are small parameters and vary in the neighborhood of the origin.

Translating $P_{*}$ to $(0,0)$ by the transformation $X=x-x_{*}$ and $Y=y-y_{*}$. Then system (43) is rewritten as

$$
\begin{aligned}
& \dot{X}=\alpha_{1} X-\alpha_{2} Y-\alpha_{3} X^{2}+\alpha_{4} X Y-\alpha_{5} Y^{2}+W_{1}(X, Y), \\
& \dot{Y}=l_{1}+l_{2} X+l_{3} Y+l_{4} X^{2}+l_{5} X Y+l_{6} Y^{2}+W_{2}(X, Y),
\end{aligned}
$$

where $W_{1}$ and $W_{2}$ are smooth functions of $X, Y$ at least of the third order. And

$$
\begin{gathered}
\alpha_{1}=\frac{\beta}{(a \beta+1)^{2}}, \quad \alpha_{2}=\frac{1}{(a \beta+1)^{2}}, \\
\alpha_{3}=\frac{(a \beta+1)^{2}[(a \beta+1)(2 m-1)+\beta]+2 a \beta^{2}}{(a \beta+1)^{2}[(a \beta+1)(2 m-1)+\beta]}, \\
\alpha_{4}=\frac{4 a \beta}{(a \beta+1)^{2}[(a \beta+1)(2 m-1)+\beta]}, \\
\alpha_{5}=\frac{2 a}{(a \beta+1)^{2}[(a \beta+1)(2 m-1)+\beta]}, \\
l_{1}=-\frac{\left[1+\mu_{1}(a \beta+1)^{2}\right][(a \beta+1)(2 m-1)+\beta] \beta \mu_{2}}{2(a \beta+1)^{3}},
\end{gathered}
$$




$$
\begin{gathered}
l_{2}=\frac{\left(1+\mu_{1}(a \beta+1)^{2}\right) \beta^{2}}{(a \beta+1)^{2}}, \\
l_{3}=-\frac{\left(1+\mu_{1}(a \beta+1)^{2}\right)\left(\beta-\mu_{2}\right)}{(a \beta+1)^{2}}, \\
l_{4}=\frac{2 \beta^{2}\left(1+\mu_{1}(a \beta+1)^{2}\right)}{(a \beta+1)[(a \beta+1)(2 m-1)+\beta]}, \\
l_{5}=-\frac{4 \beta\left(1+\mu_{1}(a \beta+1)^{2}\right)}{(a \beta+1)[(a \beta+1)(2 m-1)+\beta]}, \\
l_{6}=\frac{2\left(1+\mu_{1}(a \beta+1)^{2}\right)}{(a \beta+1)[(a \beta+1)(2 m-1)+\beta]} .
\end{gathered}
$$

Taking the change of variables $\bar{X}=X, \bar{Y}=\alpha_{1} X-\alpha_{2} Y$ $\alpha_{3} X^{2}+\alpha_{4} X Y-\alpha_{5} Y^{2}+W_{1}(X, Y)$ and rewriting $\bar{X}, \bar{Y}$ as $X, Y$, we obtain

$$
\begin{gathered}
\dot{X}=Y, \\
\dot{Y}=n_{1}+n_{2} X+n_{3} Y+n_{4} X^{2}+n_{5} X Y+n_{6} Y^{2}+W_{3}(X, Y, \mu),
\end{gathered}
$$

where

$$
\begin{aligned}
n_{1}= & -\alpha_{2} l_{1} \longrightarrow 0, \\
n_{2}= & \alpha_{4} l_{1}-\alpha_{2} l_{2}-l_{3} \alpha_{1}-\frac{2 \alpha_{5} \alpha_{1} l_{1}}{\alpha_{2}} \longrightarrow 0, \\
n_{3}= & l_{3}+\alpha_{1}+\frac{2 \alpha_{5} l_{1}}{\alpha_{2}} \longrightarrow 0, \\
n_{4}= & -l_{4} \alpha_{2}-l_{5} \alpha_{1}+l_{3} \alpha_{3}+\alpha_{4} l_{2} \\
& +\frac{-2 \alpha_{5} \alpha_{1} l_{2}+2 \alpha_{5} l_{1} \alpha_{3}-l_{6} \alpha_{1}^{2}}{\alpha_{2}} \\
& -\frac{2 \alpha_{5} l_{1} \alpha_{4} \alpha_{1}+l_{3} \alpha_{5} \alpha_{1}^{2}}{\alpha_{2}^{2}}+2 \frac{\alpha_{5}^{2} l_{1} \alpha_{1}^{2}}{\alpha_{2}^{3}} \longrightarrow-\frac{\beta}{(a \beta+1)^{2}}, \\
n_{5}= & l_{5}-2 \alpha_{3}+\frac{2 \alpha_{5} l_{2}+\alpha_{4} \alpha_{1}+2 l_{6} \alpha_{1}}{\alpha_{2}} \\
& +\frac{2 \alpha_{5} \alpha_{1} l_{3}+2 \alpha_{5} l_{1} \alpha_{4}}{\alpha_{2}^{2}}-4 \frac{\alpha_{5}^{2} l_{1} \alpha_{1}}{\alpha_{2}^{3}} \longrightarrow-2, \\
n_{6}= & -\frac{l_{6}}{\alpha_{2}}-\frac{\alpha_{4}}{\alpha_{2}}-\frac{l_{3} \alpha_{5}}{\alpha_{2}^{2}} \\
& +2 \frac{\alpha_{5}^{2} l_{1}}{\alpha_{2}^{3}} \longrightarrow-\frac{2(a \beta+1)}{(a \beta+1)(2 m-1)+\beta},
\end{aligned}
$$

with $\mu_{1} \rightarrow 0, \mu_{2} \rightarrow 0$.
Taking $u=X+n_{3} / n_{5}$, substituting $u$ in system (46), and rewriting $u$ as $X$, we get that

$$
\begin{gathered}
\dot{X}=Y, \\
\dot{Y}=n_{1}-\frac{n_{2} n_{3}}{n_{5}}+\frac{n_{4} n_{3}^{2}}{n_{5}^{2}}+\left(n_{2}-\frac{2 n_{4} n_{3}}{n_{5}}\right) X \\
+n_{4} X^{2}+n_{5} X Y+n_{6} Y^{2}+W_{4}(X, Y, \mu),
\end{gathered}
$$

where $W_{4}$ is a smooth function of $X, Y$, and $\mu$ at least of order three. When $\mu_{1} \rightarrow 0, \mu_{2} \rightarrow 0$,

$$
n_{1}-\frac{n_{2} n_{3}}{n_{5}}+\frac{n_{4} n_{3}^{2}}{n_{5}^{2}} \longrightarrow 0, \quad n_{2}-\frac{2 n_{4} n_{3}}{n_{5}} \longrightarrow 0
$$

Next, let $s=t /\left(1-n_{6} X\right), x=X$, and $y=\left(1-n_{6} X\right) Y$ into (48) and rewriting $s, x$, and $y$ as $t, X$, and $Y$ yields

$$
\begin{gathered}
\dot{X}=Y, \\
\dot{Y}=\varepsilon_{1}+\varepsilon_{2} X+n_{5} X Y+\varepsilon_{3} X^{2}+W_{5}(X, Y, \mu),
\end{gathered}
$$

where $W_{5}$ is a smooth function of $X, Y$, and $\mu$ at least of order three and

$$
\begin{aligned}
& \varepsilon_{1}=n_{1}-\frac{n_{2} n_{3}}{n_{5}}+\frac{n_{4} n_{3}^{2}}{n_{5}^{2}} \longrightarrow 0, \\
& \varepsilon_{2}=n_{2}-2 \frac{n_{4} n_{3}}{n_{5}}-2 n_{6} \varepsilon_{1} \longrightarrow 0, \\
& \varepsilon_{3}=n_{4}-2 n_{6}\left(n_{2}-2 \frac{n_{4} n_{3}}{n_{5}}\right)+n_{6}^{2} \varepsilon_{1} \longrightarrow-\frac{1}{(a \beta+1)^{2}}<0,
\end{aligned}
$$

when $\mu_{1} \rightarrow 0, \mu_{2} \rightarrow 0$. Let $x=\left(n_{5}^{2} / \varepsilon_{3}\right) X, y=\left(n_{5}^{3} / \varepsilon_{3}^{2}\right) Y$, $v=\left(\varepsilon_{3} / n_{5}\right) t$, and rewrite $v$ as $t$. Then system (50) becomes

$$
\begin{gathered}
\dot{x}=y, \\
\dot{y}=\tau_{1}+\tau_{2} x+x y+x^{2}+W_{6}(x, y, \mu),
\end{gathered}
$$

where $W_{6}$ is a smooth function of $x, y$, and $\mu$ at least of order three and $\tau_{1}=n_{5}^{4} \varepsilon_{1} / \varepsilon_{3}^{3}, \tau_{2}=n_{5}^{2} \varepsilon_{2} / \varepsilon_{3}^{2}$.

Then system (4) exists the following bifurcation curves in a small neighborhood of the origin in the $\left(\mu_{1}, \mu_{2}\right)$ plane.

Theorem 12. Let $0<m<1 / 2-\beta / 2(a \beta+1), \delta=1 /(a \beta+1)^{2}$, $h=(1 / 4)(\beta /(a \beta+1)-1)^{2}+m \beta /(a \beta+1)$. Then system $(43)$ admits the following bifurcations:

(i) there exists a saddle node bifurcation curve $S N=$ $\left\{\left(\mu_{1}, \mu_{2}\right): 4 \varepsilon_{3} \varepsilon_{1}=\varepsilon_{2}^{2}+o(\|\mu\|)^{2}\right\} ;$

(ii) there is a Hopf bifurcation curve $H=\left\{\left(\mu_{1}, \mu_{2}\right): \varepsilon_{1}=\right.$ $\left.0+o(\|\mu\|)^{2}, \quad \varepsilon_{2}<0\right\}$

(iii) there is a homoclinic bifurcation curve $H L=\left\{\left(\mu_{1}, \mu_{2}\right)\right.$ : $\left.25 \varepsilon_{1} \varepsilon_{3}+6 \varepsilon_{2}^{2}=0+o(\|\mu\|)^{2}\right\}$. 
The biological interpretation for the Bogdanov-Takens bifurcation is that if the harvesting rate $h$ and the prey refuge value $m$ satisfy $0<m<1 / 2-\beta / 2(a \beta+1), h=$ $(1 / 4)(\beta /(a \beta+1)-1)^{2}+m \beta /(a \beta+1)$, and $\delta=1 /(a \beta+1)^{2}$, then the predator and prey coexist in the form of a positive equilibrium or a periodic orbit for different initial values, respectively. And there exist other values of parameters, such that the predator and prey coexist in the form of a positive equilibrium for all initial values lying inside the homoclinic loop, and the predator and prey coexist in the form of a periodic orbit with infinite period for all initial values on the homoclinic loop. By choosing $\beta=2, a=1.6, m=$ $(1 / 4)(1-\beta /(a \beta+1)), h=(1 / 4)(\beta /(a \beta+1)-1)^{2}+m \beta /(a \beta+$ $1)$, and $\delta=1 /(a \beta+1)^{2}$, the numerical simulations for the Bogdanov-Takens bifurcation in Theorem 12 can be shown in Figures 3, 4 and 5.

3.5. Separatrix Connecting a Saddle-Node and a Saddle Bifurcation and Heteroclinic Bifurcation. From Theorem 8 and Lemma 3, when $0<m<(1 / 2)(1-\beta /(a \beta+1))$, $h=\tilde{h}, \delta \neq 1 /(a \beta+1)^{2}$, there may exist a separatrix connecting the saddle-node $P_{*}$ and the saddle $E_{1}$. When $0<m<(1 / 2)(1-\beta /(a \beta+1)), m(1-m)<h<$ $\tilde{h}$, the saddle node $P_{*}$ separates into the hyperbolic node $P_{1}$ and the hyperbolic saddle $P_{2}$, which implies that system (4) undergoes a separatrix connecting a saddle node and a saddle bifurcation. Furthermore, the heteroclinic bifurcation may occur if there exists a heteroclinic orbit connecting the separatrix of saddle $E_{1}$ and saddle $P_{2}$.

\section{Acknowledgments}

This paper is supported by NSFC (11226142), Foundation of Henan Educational Committee (2012A110012), Foundation of Henan Normal University (2011QK04, 2012PL03), Natural Science Foundation of Shanghai (12ZR1421600), and Shanghai Municipal Educational Committee (10YZ74).

\section{References}

[1] Z. Liang and H. Pan, "Qualitative analysis of a ratio-dependent Holling-Tanner model," Journal of Mathematical Analysis and Applications, vol. 334, no. 2, pp. 954-964, 2007.

[2] J. Rebaza, "Dynamics of prey threshold harvesting and refuge," Journal of Computational and Applied Mathematics, vol. 236, no. 7, pp. 1743-1752, 2012.

[3] R.P. Gupta and P. Chandra, "Bifurcation analysis of modified Leslie-Gower predator-prey model with Michaelis-Menten type prey harvesting," Journal of Mathematical Analysis and Applications, vol. 398, no. 1, pp. 278-295, 2013.

[4] X. Liu and M. Han, "Chaos and Hopf bifurcation analysis for a two species predator-prey system with prey refuge and diffusion," Nonlinear Analysis, vol. 12, no. 2, pp. 1047-1061, 2011.

[5] Z. Ma, W. Li, Y. Zhao, W. Wang, H. Zhang, and Z. Li, "Effects of prey refuges on a predator-prey model with a class of functional responses: the role of refuges," Mathematical Biosciences, vol. 218, no. 2, pp. 73-79, 2009.

[6] L. Chen, F. Chen, and L. Chen, "Qualitative analysis of a predator-prey model with Holling type II functional response incorporating a constant prey refuge," Nonlinear Analysis, vol. 11, no. 1, pp. 246-252, 2010.

[7] D. Xiao and L. S. Jennings, "Bifurcations of a ratio-dependent predator-prey system with constant rate harvesting," SIAM Journal on Applied Mathematics, vol. 65, no. 3, pp. 737-753, 2005.

[8] D. Xiao, W. Li, and M. Han, "Dynamics in a ratio-dependent predator-prey model with predator harvesting," Journal of Mathematical Analysis and Applications, vol. 324, no. 1, pp. 1429, 2006.

[9] L.-L. Wang, Y.-H. Fan, and W.-T. Li, "Multiple bifurcations in a predator-prey system with monotonic functional response," Applied Mathematics and Computation, vol. 172, no. 2, pp. 11031120, 2006.

[10] Y. Tao, X. Wang, and X. Song, "Effect of prey refuge on a harvested predator-prey model with generalized functional response," Communications in Nonlinear Science and Numerical Simulation, vol. 16, no. 2, pp. 1052-1059, 2011.

[11] F. S. Berezovskaya, B. Song, and C. Castillo-Chavez, "Role of prey dispersal and refuges on predator-prey dynamics," SIAM Journal on Applied Mathematics, vol. 70, no. 6, pp. 1821-1839, 2010. 


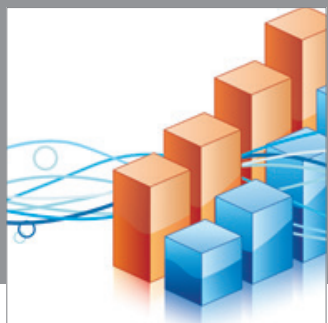

Advances in

Operations Research

mansans

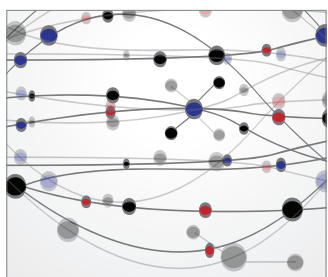

The Scientific World Journal
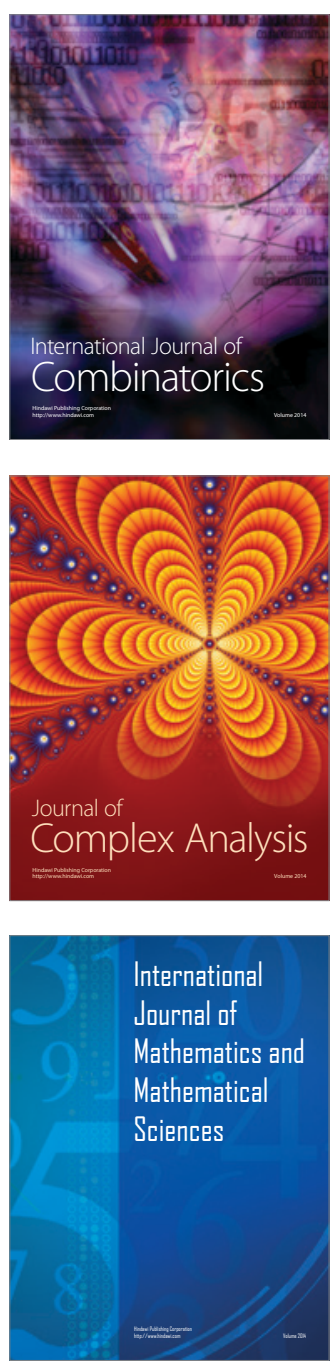
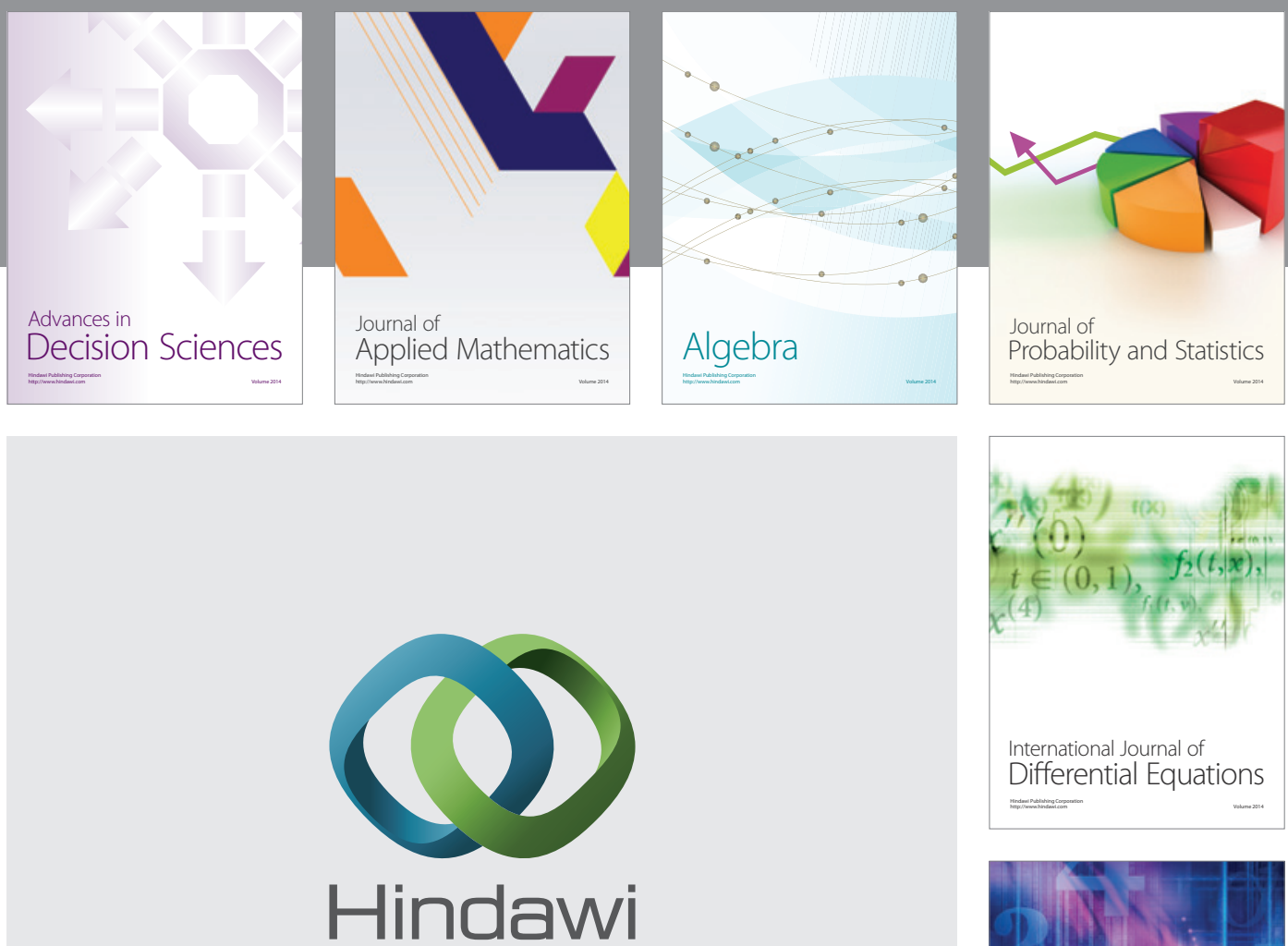

Submit your manuscripts at http://www.hindawi.com
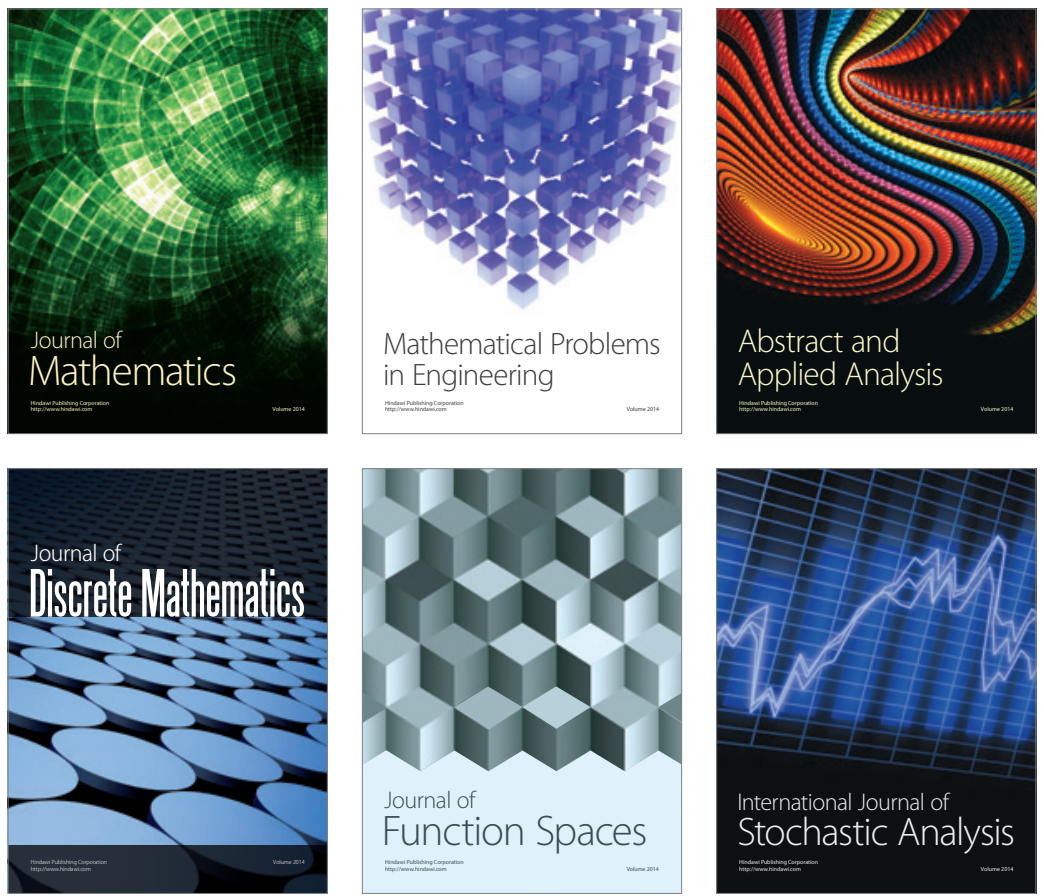

Journal of

Function Spaces

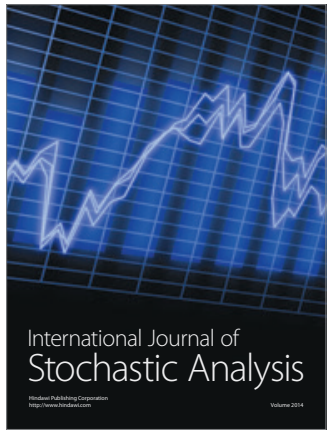

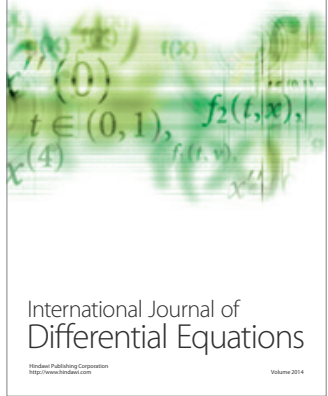
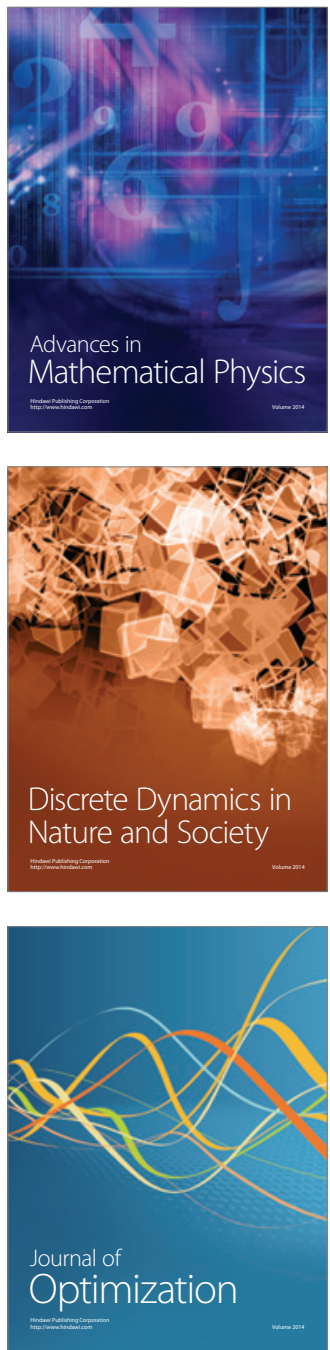